\title{
PRENDAS NeCESARIAS DE LAS MUJERES PARA EL OfICIO DE PARTEAR: EL MANUAL DEL PROTOMEDICATO
}

\author{
Mónica Adriana Luna-Blanco
}

L as mujeres cotidianamente están pariendo al mundo desde sus cuerpos y sus realidades socioculturales. El oficio de las parteras se puede considerar como una de las primeras profesiones, y el reconocimiento de sus prácticas en la escala social ha variado en los distintos momentos históricos: desde el prestigio, hasta la exclusión e invisibilización. A partir del siglo XIX, la partería se profesionalizó a través de la enfermería como un oficio femenino bajo el resguardo de la medicina masculina.

En el acto de parir se entrecruzan la etnia, la clase, la raza, el género, la edad y las políticas públicas y religiosas, así como las dinámicas propias de cada cultura y los contextos que éstas ofrecen para las mujeres. En dichos contextos, las parteras han desempeñado un papel central como actoras en el proceso del embarazo, el alumbramiento y el puerperio; más aún cuando las mujeres eran quienes asistían de forma exclusiva a otras mujeres en los eventos de nacimiento, esto es, antes de que se institucionalizaran las prácticas médicas hegemónicas (Conde, 2011).

En los tiempos, culturas y pueblos de todo el mundo, las parteras han asistido partos desde en las habitaciones de reinas - donde eran bien remuneradas por su trabajo-, hasta en sencillos cuartos de las localidades más apartadas, donde demostraban sus conocimientos empíricos sobre herbolaria, los ciclos de gestación y los cuerpos femeninos. Con el devenir del tiempo, las parteras adquirieron destrezas manuales y un desarrollo del sentido de tacto sobre la anatomía femenina y el feto dentro del útero, siendo ellas, en muchos casos, las únicas personas cercanas y con posibilidades para atender a otras mujeres en el trabajo de parto.

Destaca que, en la transmisión de esta práctica, el cúmulo de conocimientos adquiridos se transmitía de generación a generación, de mujer a mujer, de forma oral, a través de una práctica cotidiana y cercana entre parteras y aprendices, y de ambas con los cuerpos de las mujeres a quienes procuraban la atención, de tal forma que el conocimiento empírico se reelaboraba a partir de los casos que unas y otras iban resolviendo (Gallego et al., 2005). Con la suma de todo ello, las parteras alcanzaron un amplio conocimiento sobre la anatomía femenina y los ciclos del embarazo, y lograban manipular de formas adecuadas los vientres, y a las criaturas dentro de ellas, para lograr el alumbramiento. Así, las parteras se vinculaban directamente a través de su oficio con la reproducción social (Luna, Sánchez y Velasco, 2015).

\footnotetext{
Mónica Adriana Luna Blanco. Doctora en Ciencias en Ecología y Desarrollo Sustentable por El Colegio de la Frontera Sur, México. Catedrática Conacyt en el Centro de Estudios Superiores de México y Centroamérica de la Universidad de Ciencias y Artes de Chiapas, México. Correo electrónico: lilithssa08@gmail.com.
}

Recibido: 11 de octubre de 2017. 
Al iniciarse las profesionalizaciones de las ciencias médicas en los siglos XIV y XIX, ${ }^{1}$ estas habilidades, sabidurías y acciones concretas que realizaban las parterasfueronidentificadas como elementos que debían ser controlados por los varones, y las especialidades médicas como la ginecología y la obstetricia iniciaron un proceso de apropiación de sus saberes, así como un desplazamiento del lugar central que las parteras ocupaban en la atención al parto, de manera que colocaron la práctica de la partería en un lugar de subordinación de la mujer frente a una ciencia médica patriarcal. La religión jugó un papel preponderante en dicho desplazamiento y en el control de la partería por dos motivos principales. El primero de ellos era que las parteras, por su contacto íntimo y cercano con las mujeres y sus procesos reproductivos y sexuales, no se limitaban al acompañamiento del embarazo y a la atención del parto, sino que también a ellas acudían mujeres que deseaban interrumpir su embarazo, siendo el aborto una acción considerada pecado capital, la Iglesia necesitaba impedir que las parteras realizaran este tipo de prácticas. El segundo motivo consistía en que, al ser las parteras quienes recibían al recién nacido, y en caso de que éste no lograra sobrevivir, ellas podían impartir el bautismo y de ese modo evitar que el alma de ese infante quedara atrapada en el limbo. Las parteras, entonces, debían poseer, demostrar y cumplir con cualidades físicas, morales y prácticas de exploración muy específicas sobre los cuerpos femeninos, exigidas por el gremio médico y por la Iglesia. Las parteras "necesitaban" ser aprobadas para ejercer su oficio. Una de las formas en que se concretó ese proceso de auscultación fue a través de la elaboración de manuales de capacitación, entre los que se encuentra la Cartilla Nueva, útil y necesaria para instruirse las Matronas, que vulgarmente se llaman Comadres, en el oficio de Partear, el cual se presenta en este texto.

Antes de entrar en el tema, cabe mencionar que en ese ambiente de búsqueda de control de la labor de las parteras, en el marco de la formación teórico-práctica de los oficios en la España virreinal, se decidió que las matronas debían pasar por procesos en los que se examinaran y validaran sus capacidades para tal labor, y era el Real Tribunal del Protomedicato, ${ }^{2}$ instaurado por los reyes católicos en el siglo $\mathrm{XV}$, la figura encargada de tal examinación (Gallego et al., 2005). Un primer intento de plasmar por escrito la labor de la parteria fue en el año 1541, cuando se editó en España el libro Arte de Las Comadres o Madrina, del Regimiento de las Preñadas y Paridas y de los niños, redactado por el médico Damía Carbó (Manual de Carbó). Este texto contiene información médica sobre obstetricia, ginecología y pediatría desde una visión medieval; por ejemplo, en él se lee cómo en esa época aún se consideraba que sólo las mujeres podían encargarse de ese tipo de labores ya que eran inadecuadas, "cosas feas", para ser realizadas por los hombres. Lo interesante es que el libro, escrito por un médico hombre, está destinado a las comadronas para que éstas adquieran conocimientos acerca de su oficio como "cosa de mujeres". Magdalena Santo Tomás (2001) identifica como elemento de gran interés cómo en el manual se refleja la fundamentación médica existente en el siglo XVI y el papel tradicional que desempeñaban las mujeres en relación con el parto y la atención al recién nacido; sobre todo, en el texto se reconoce la partería como una labor femenina, pero con una gran falta de conocimiento sobre los fundamentos en los que las parteras apoyaban su práctica (Santo Tomás, 2001: 237-238). Ante estas supuestas carencias en las parteras, se posicionó el conocimiento médico masculino como el único poseedor de las prácticas y teorías médicas necesarias y permitidas para la atención del parto.

Doscientos años después, en 1750, se publicó la Cartilla Nueva, útil y necesaria para instruirse las Matronas, quevulgarmente se llaman Comadres, en el oficio de Partear, que mandó redactar el Real Tribunal del Protomedicato al doctor Antonio Medina. Este texto no sólo se utilizó en España, sino que llegó hasta tierras virreinales en México. El objetivo central de esta cartilla era vigilar, examinar y reemplazar a las parteras tradicionales —quienes comúnmente eran mujeres sin alfabetizar, y, en el caso de México, también indígenas- por "mujeres alfabetas entrenadas por los médicos, que permitiesen a éstos el acceso a embarazadas, parturientas y puérperas" (Carrillo, 1999: 170). 
La cartilla se compone de 89 páginas. Inicia con un prólogo al que siguen cuatro capítulos: Capítulo I, Proemial; Capítulo II, De la Anatomía; Capítulo III, Del Estado de Preñez; Capítulo IV, En que se trata del parto.

En el prólogo se hace evidente la visión judeocristiana bajo la cual la sociedad se desenvolvía ya que, además de citar a las parteras y su labor en los textos del Antiguo Testamento, se hace mención a la condición destinada a las mujeres - a partir del pecado original — de parir a sus hijos con dolor, riesgo y trabajos. Al ser inevitable esta circunstancia, se menciona que son las matronas las encargadas de dar consuelo y esperanza en dicho trance.

Al igual que en el Manual de Carbó, esta cartilla es una ventana de conocimiento sobre las formas en que se entendían en esa época procesos como la reproducción, la formación y la función de la placenta, e incluso los embarazos molares y sus posibles causas. Destacan en la obra las formas de nombrar los órganos genitales femeninos y sus componentes - "nymphas", "vagina del útero", "hymen", "clítoris"-, y los términos relacionados con la fecundación y el proceso de gestación - "fetus", "membranas del fetus", "licor en el que nada el fetus", "placentas"- La última parte se enfoca en los cuidados que la matrona debía proveer al recién nacido.

A la par se reconoce el oficio de partear como exclusivo de mujeres; no obstante, "debido al abuso, la impericia y desconfianza de ellas", es necesario reconocer que en casos de parto difícil "se debe recurrir a la destreza superior de un buen cirujano". Esta diferencia entre la destreza superior y la impericia de las parteras en sus labores se menciona a lo largo de los cuatro capítulos. En específico, se señala como obligación de las parteras reconocer sus debilidades y carencias, y por ello, ante casos de partos complicados, debían acudir a un cirujano. Así, se establece que todas las mujeres que se dedicaran a ese oficio debían ser examinadas por el Tribunal del Protomedicato, para lo cual tenían que recibir una capacitación teórica que las preparase para responder de forma adecuada a sus interrogatorios, de ahí que los capítulos se presenten a manera de cuestionario: iqué se debe entender por "arte de partear"? , ¿cúal es el sujeto que lo debe ejercitar?, ¿qué prendas deberá tener la matrona para profesar el arte de partear?
Nos detendremos en específico en esta última pregunta, ya que resulta interesante cómo el afán de control del Tribunal, cuyo examen a las matronas implicaba la revisión tanto de aspectos de su fisionomía, como de sus virtudes morales, entre las que debían destacar la obediencia, la fe incuestionable en Dios, la misericordia hacia el prójimo, y un genio dócil que le permitiera "admitir el dictamen de sus mayores en suficiencia" (p. 6).

En el texto se establecen diferencias en los niveles de conocimiento teórico que ellas podían adquirir a diferencia de los profesores: basta con que sus saberes sobre la anatomía femenina sean superficiales. El manual busca también desmitificar creencias sobre las parteras y sobre las mujeres en general; por ejemplo, que el útero está libre y suelto y por ello puede tener movimiento. Se advierte además que el aborto — tanto practicarlo como solicitar que sea realizado - es causa de excomunión.

El manual hace énfasis en la imperiosa necesidad de que, ante eventos considerados por los médicos como de riesgo en el parto, o ante una situación de muerte fetal, las matronas deben avisar y acudir inmediatamente al cirujano "para que consultado el verdadero juicio que de sebe hacer, den las providencias para el remedio; pues este caso trae dificultades, que nos las puede superar la matrona, y complicación de accidentes, en que no tiene la inteligencia necesaria" (p. 47).

El origen de la profesionalización del oficio de las parteras se especifica tanto en el manual de Carbó, como en la cartilla que nos ocupa, al menos para el caso de España y de México, y con ello la expropiación de su conocimiento, de sus habilidades y, por último, de su oficio (Herrera, 1997; Carrillo, 1999; Gallego et al., 2005; Serrano, 2015, entre otros). Si se lee con detenimiento la Cartilla Nueva, útil y necesaria para instruirse las Matronas..., quienes están cercanos a las problemáticas actuales en que se desarrolla la labor de la partería en México —en zonas indígenas, urbanas, rurales, mestizas - podrán sin duda identificar las similitudes entre el texto de esta cartilla y los discursos médicos actuales, en los que se sitúa a las parteras como subalternas, carentes de sabiduría y de conocimientos adecuados para hacer aquello de lo que 
como mujeres nos hemos hecho cargo desde los primeros tiempos de la humanidad: acompañar y atender los procesos de alumbramiento. Seguimos frente al reto social de entender que las bondades biomédicas desarrolladas en los siglos XX y XXI permiten actuar a favor de las vidas de las mujeres y de sus crías en casos de emergencia obstétrica, y eso no debería demeritar ni descalificar la labor milenaria de las parteras, quienes en no pocos lugares continúan siendo la única opción de atención en el parto, o se están constituyendo como otra opción posible para que las mujeres no pasen por un embarazo medicalizado y experimenten un parto respetado.

A continuación se presenta la transcripción del capítulo Proemial de la Cartilla Nueva, útil y necesaria para instruirse las Matronas, que vulgarmente se llaman Comadres, en el oficio de Partear. Mandada hacer por el Real Tribunal del Protho-Medicato al doctor don Antonio Medina, que trata sobre el parto.

\section{Notas}

${ }^{1}$ Serrano (2015) da cuenta cómo en España en el siglo XIV los médicos graduados en universidades comenzaron a atacar las prácticas de las matronas cuestionando sus remedios y prácticas curativas. Más adelante, en el siglo XIX, la autora plantea cómo la profesión médica "exclusivamente masculina" y con mayor poder, intentó hacerse cargo exclusivo de la asistencia al parto. Para el caso de México, Carrillo (1999) aporta un panorama completo sobre dicha profesionalización.

${ }^{2}$ Este tribunal, nacido en el siglo XV en España, tuvo como finalidad regular el ejercicio de la medicina. Estaba conformado por protomédicos y examinadores que reconocíanlasuficienciadequienes aspiraban asermédicos y concedía las licencias necesarias para el ejercicio de dicha profesión. Es la primera institución de administración de la salud pública española (Álvarez, 2008).

\section{Referencias}

Álvarez Vázquez, Jorge (2008). Diccionario de historia de la medicina [documento inédito].
Carrillo Ana. M. (1999). "Nacimiento y muerte de una profesión. Las parteras tituladas en México". En DYNAMIS, Acta Hispanica ad Medicinae Scientiarumque Historiam Illustrandam, 19: 167-190.

Conde, F. (2011). "Parteras, comadres, matronas. Evolución de la profesión desde el saber popular al conocimiento científico". En Discursos Académicos, 49. España: Academia de Ciencias e Ingenierías de Lanzarote.

Gallego Caminero, Gloria, Margalida Miró Bonet, Pilar Ferrer de Sant Jord y Denise Gastaldo (2005). "Las parteras y/o comadronas del siglo XVI: el manual de Damiá Carbó". En Texto \& Contexto - Enfermagem, 14(4): 601-607. Disponible en: http://www.scielo. $\mathrm{br} /$ scielo.php?script=sci_arttext\&pid=S010407072005000400018\&lng=es\&tlng=es.

Herrera, R.F. (1997). "Un manual para la formación de las comadronas españolas (1929)”. En Revista Cultura de los Cuidados, I(1): 37-42.

Luna Blanco, Mónica, Georgina Sánchez Ramírez y S.J. Velasco (2015). "Parteras institucionalizadas en San Cristóbal de las Casas: su labor a lo largo de tres décadas". En Georgina Sánchez Ramírez (coord.), Imagen instantánea de la partería. México: El Colegio de La Frontera Sur, Asociación Mexicana de Partería, pp. 49-84.

Medina, A. (1785). Cartilla Nueva, útil y necesaria para instruirse las Matronas, que vulgarmente se llaman Comadres, en el oficio de Partear. Mandada hacer por el Real Tribunal del Protho-Medicato al doctor don Antonio Medina. Disponible en:http://www.cervantesvirtual.com/obra/ cartilla-nueva-util-y-necesaria-para-instruirse-lasmatronas-que-vulgarmente-llaman-comadres-en-eloficio-de-partear.

Santo Tomás Pérez, Magdalena (2001). "Reseña de: Carbón, Damián, Libro del arte de las comadres o madrinas $y$ del regimiento de las preñadas y paridas y de los niños". En Edad Media. Revista de Historia, 4: 236-238.

Serrano, M.I. (2015). "Apuntes históricos de la profesión de matrona". En Matronas y mujeres en la historia. Pamplona, España: Asociación Navarra de Matronas, pp. 1-12. Disponible en: http://matronasdenavarra. com/wpcontent/uploads/2015/04/historia.pdf. 


\title{
Cartilla Nueva, útil y necesaria para instruirse las Matronas, que vulgarmente se llaman Comadres, en el oficio de Partear.
}

\author{
Mandada Hacer por el Real Tribunal del ProtoMedicato, al Doctor Don Antonio Medina, Médico de los Reales \\ Hospitales, de la Real Familia de la Reyna nuestra Señora, \\ y Examinador del mismo Tribunal. \\ Con las licencias Necesarias. En Madrid en Casa de Antonio Delgado, año de 1785.
}

\section{Capitulo I. Proemia}

P. ¿Qué prendas deberá tener una Matrona para profesar el Arte de Partear?

R. Lo primero, ha de emprender este ejercicio cuando se halla en una edad proporcionada, para poder emplear el espacio de algunos años en la Teórica, y regLas de la Práctica; porque si cuando ya vieja lo intenta, se le ha pasado el tiempo oportuno de aprender, su entendimiento y sus sentidos se hallan ya débiles para ello, y sus corporales fuerzas insuficientes.

Lo segundo, no se deben admitir a este ejercicio las muy jóvenes, ni las doncellas, sino es el caso de destinarse por suficiente tiempo, desde esta edad, al lado de Matronas hábiles o de cirujanos proáctivos en el Arte, a habilitarse en todo lo necesario para obrar por sí.

Lo tercero, deben tener una salud robusta; porque si son débiles, o enfermizas, no pueden tolerar el trabajo de trasnochar, y demás incomodidades corporales inseparabes del ejercicio, ni tener las fuerzas necesarias para las varias maniobras que se ofrecen, por cuyos motivos son también inhábiles las excesivamente gordas.

Lo cuarto, no ha de tener la mano callosa, ni disformemente grande, porque con la introducción en el orificio angosto y delicado excitan intensos dolores y ofensas muy perjudiciales: como asismismo no ha de tener los dedos, o el brazo encorbados, o convulsos, ni con otro algún vicio de los que quitan, o debilitan el sentido del tacto.

Lo quinto, ha de saber leer, y escribir suficientemente, y ha de tener entendimiento capaz de aprender lo que sus Maestros la enseñen, y de aprovechar, por la lección de los Libros, lo necesario para tener una felíz práctica.

Lo sexto, debe ser vigilante y cuidadosa; pues no pocas veces por la pereza, y tardanza se pasa la ocasión, y se pone la madre, y el infante en gravísimo riesgo.

Lo séptimo; debe ser de genio dócil, y propenso a admitir el dictamen de sus mayores en suficiencia, pidiendo en oportuno tiempo el auxilio, y la consulta, ya del Médico, ya del Cirujano, en los casos en que ocurra cualquier dificultad: lo que obtendrá fácilmemte siempre que de sí propia no tenga aquella satisfacción, que comúnmente tienen las ignorantes.

Lo octavo, debe ser misericordiosa, y tan igual en ocurrir a las necesidades de los pobres, como a las asistencias de los ricos; porque Dios, que a su imagen, y semejanza hizo iguales las Almas, cuida más de recompensar las misericordias con los necesitados, que los poderosos del mundo a sus sirvientes.

Lo noveno, debe ser buena cristiana y confiada mas en los auxilios de Dios, que en los de su Arte; pues devotamente implorados, los concede su Divina Providencia suficientes para salir del mayor aprieto. 
Lo décimo, debe ser de ánimo benigno, paciente, y alegre con modestia; porque la pusilanimidad de las Parturientas se depone con estos agradables afectos, y recobradas en su natural valor se ayudan para el buen éxito.

Lo undécimo, debe ser templada, con especialidad en el uso del vino; pues de lo contrario se constituyen inútiles y perjudiciales.
Lo duodécimo, debe ser fiel, y silenciosa; porque de faltar al secreto en los casos en que importa, se sigue infamia, y deshonor.

Finalmente, otras virtudes, asi de cuerpo como de alma, les son propias (aunque no totalmente indispensables) a las que deben ejercitar este Arte; pero no se expresan, porque no son tan escenciales como las dichas. 


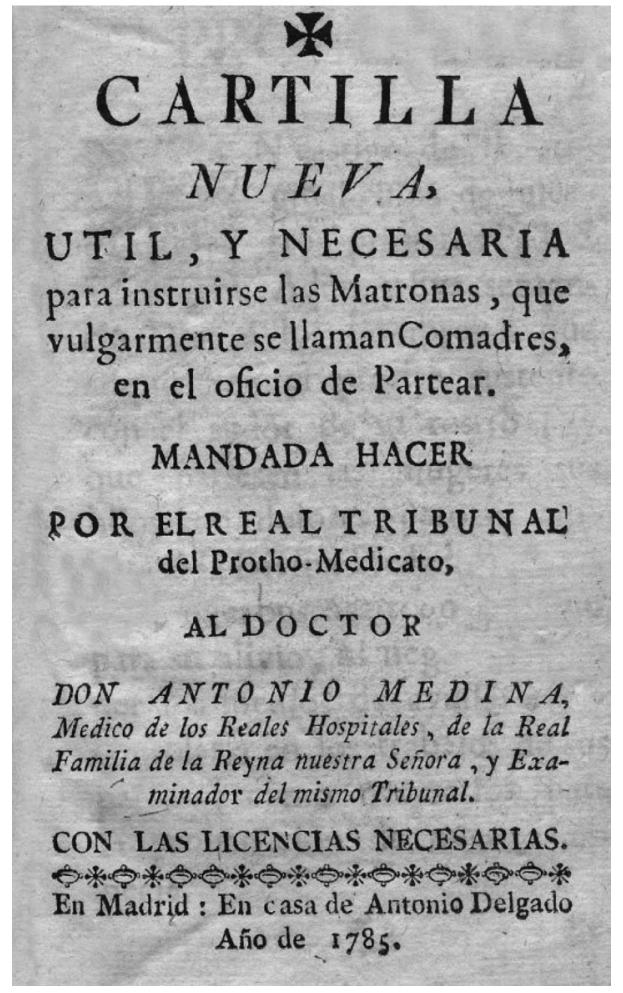

2 Cartilla nueva, yutil

P. Qual es el objeto de este Arte?

R. La muger en el estado de preñéz, hasta el fin de su puerperio, ó sobreparto.

P. Qual es el sugeto que lo debe exercitar?

R. Pơr lo regular, y comun una Marrona, en quien concurran da's hecesarias prendas; pues aunque no se pueden excluir de este exercicio los Girujanos, que Haman vulgarmente Comadrones; los debe reservaren da honesti, dado y y decencia para los casos unicamente en que ocurre dificultad insuperable por la $\mathrm{Ma}_{5}$ trona: la qual dificultad no es tan frequente, come la vana .9 ti-

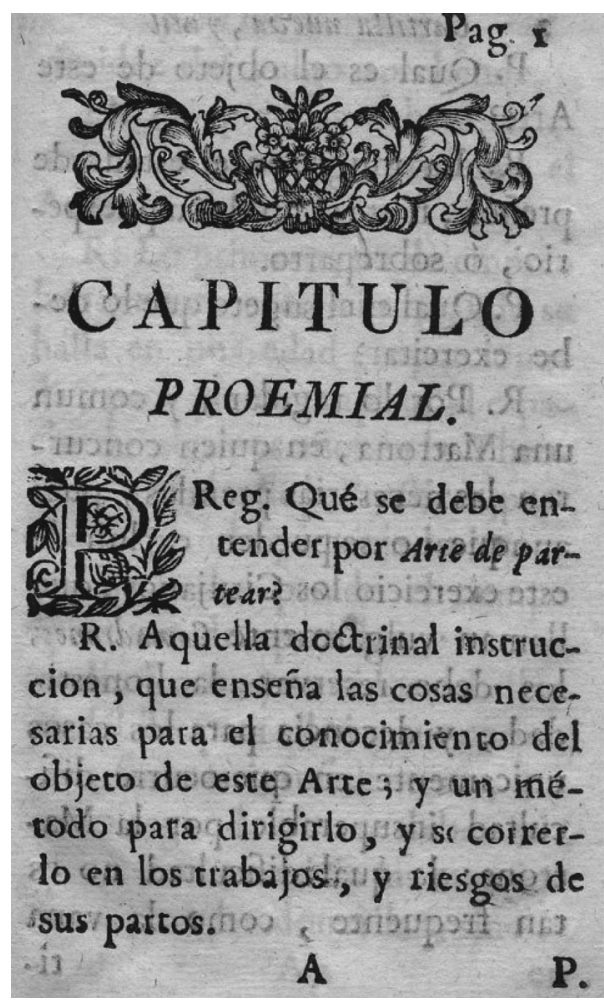

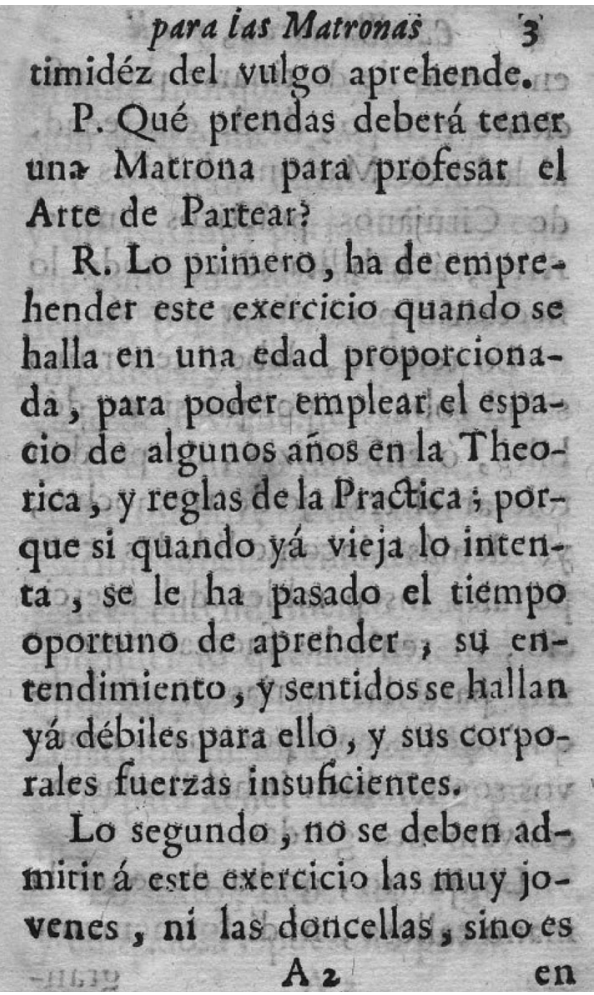




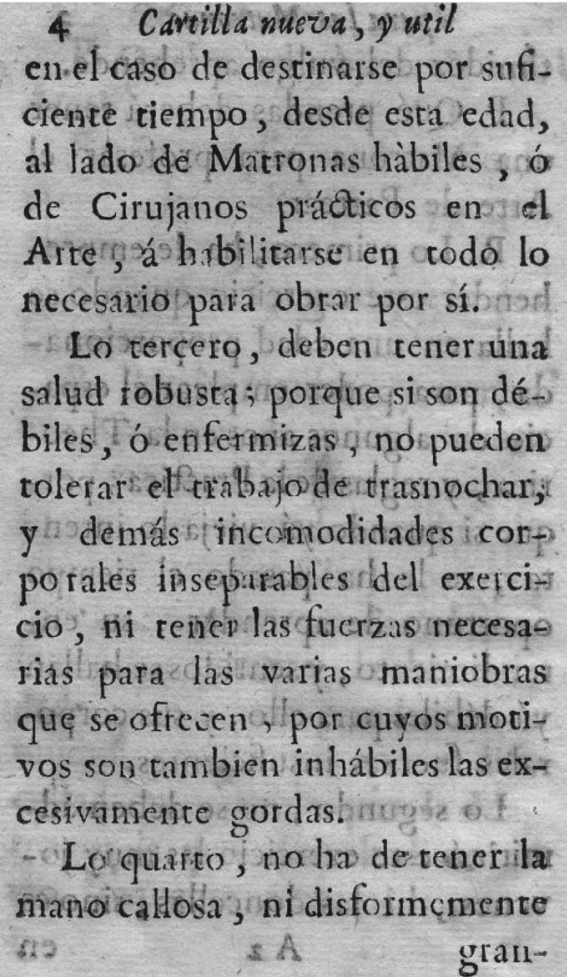

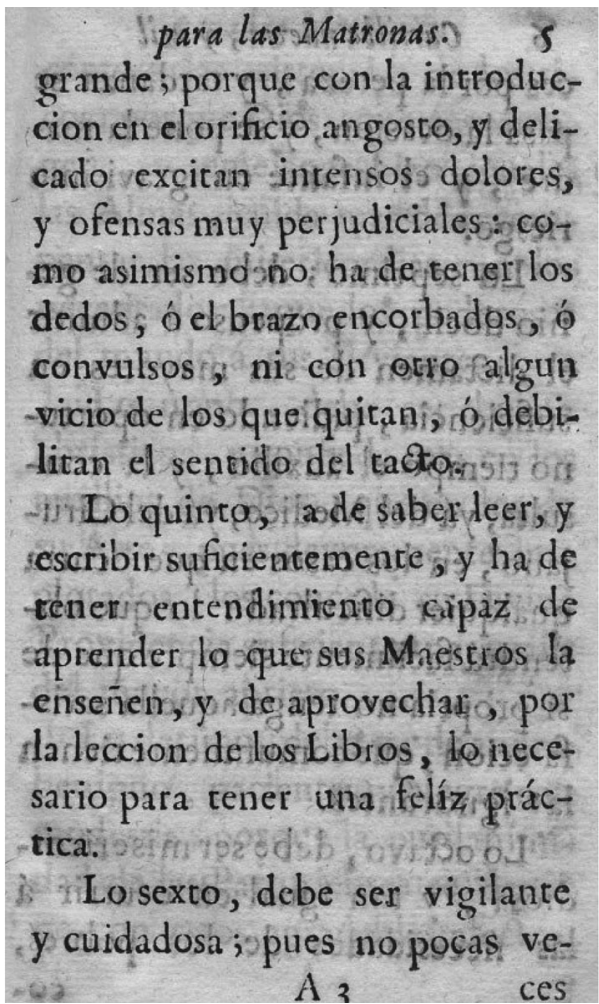

Jara las Matronas. $\delta$ grande ; porque conla introduccion en el orificio angosto, y delicado excitan intensos dolores, y ofensas muy perjudiciales: $\mathrm{Ca}_{-}$ mo asimismd nó harde tenerlos dedos, ó el brazo encorbados, $\theta$ convulsos, ni con orto algun -vicio de los queíquitani, ió debilitan el sentido del tactomisis on -in Lo quinto, iade saber leer, y escribir suficieatemente, y ha de rener entendimiento capaz de aprender losque sus Maestros la enseñen, y de aprovechiaro por da leccion de los bibros, lonecesario para tener una felízipráctica. is tho sexto, debe ser vigilante y cuidadosa ; pues no pocas veA 2

6 Cartillanuern, yntil ces por la pereza, y tardanza se pasa la ocasion, y se pone la ma. die, y el infante en gravisimo riesgo. 20. Lo seprima; debe ser de ger nio docil a y propenso á admitir el dictamen de sus mayores en suficiençia, pidiendo en oportuno riempo el auxilio, y la consulta, yá del Medico, yá del Cirujano, en los casos en que iocura qualquier dificultad: la que obtendrá facilmente siempre que de sí proprib no tenga aquella satis$\mathrm{fa}$ ccion, que comunmente tieneh las ignorantes. 79153 sisq oima

Lo adtavo, debesermisericordiosatigiy tan igual en ocuryir á las necesidades de los pobres,

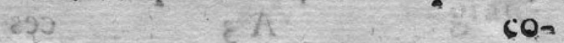




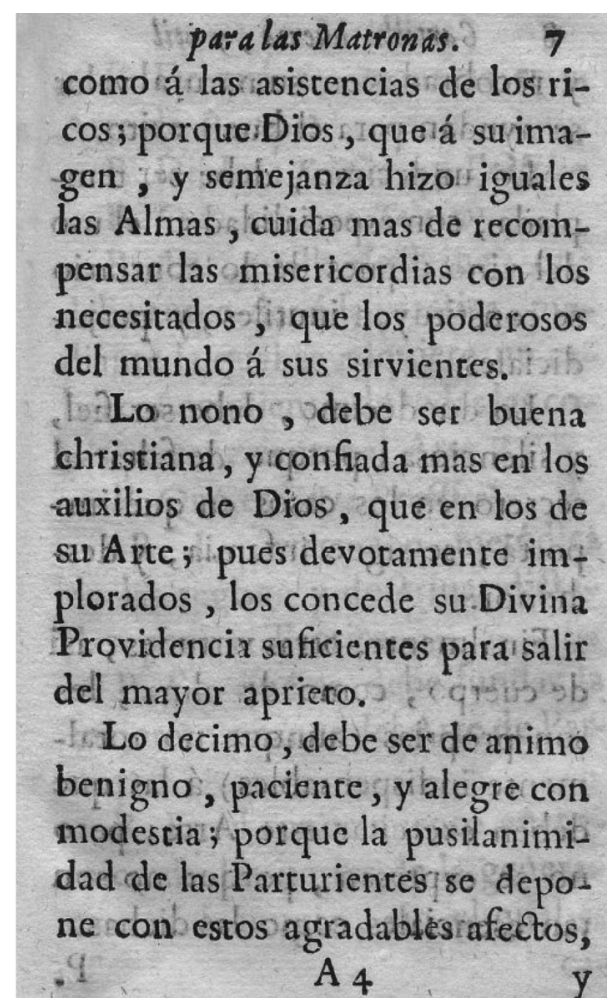

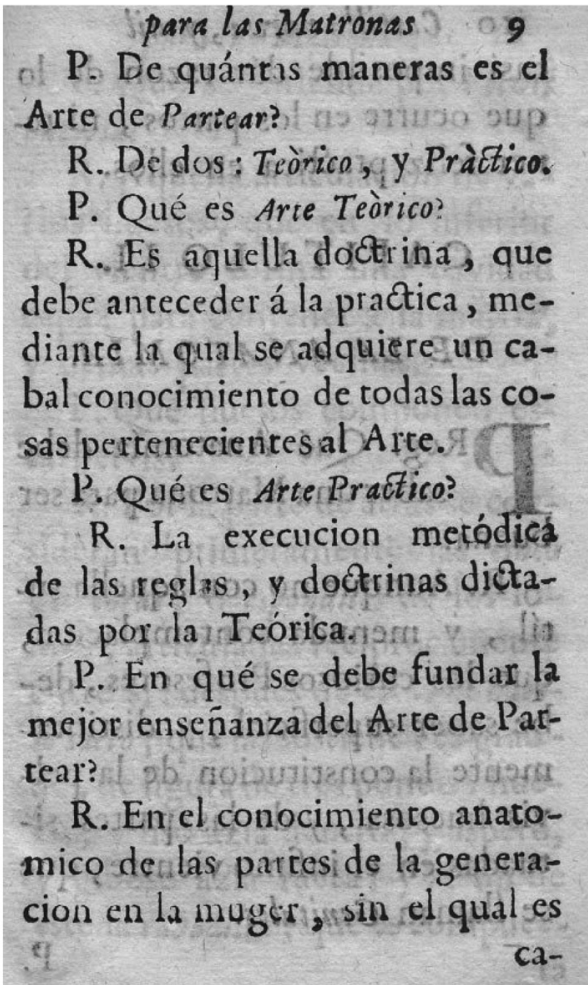

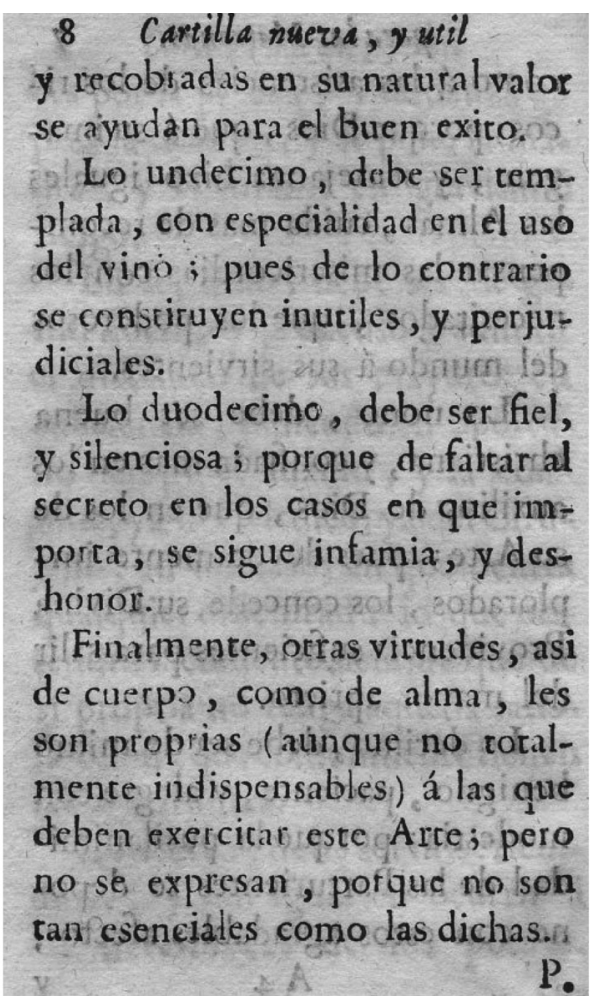

Io Cartilla nueva, y util

casi imposible dár razon de lo que ocurre en los partos, ni tener felíz practica en ellos.

SLO CA PIT ULO II.

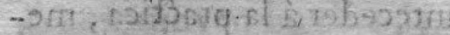

-5) DE LAPA NATOMIA.

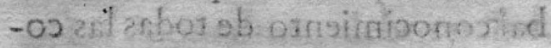
Deg. Qué Aniatomía debe saber una Matrona para ser Eüena?

$-f R$. Aunque no con aquella sutíl, y menada contemplacion, eque los curiosos Profesores, debe saber superficial, y distintamente la constitucion de la pelvis huesosa, y de las partes situadas en el infimo vientre, que

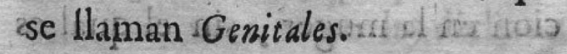

$-53$ P. 